OPEN ACCESS

Edited by:

Rajko Reljic

St George's, University of London,

United Kingdom

Reviewed by:

Pietro Speziale,

University of Pavia, Italy

Peter Timmerman

Pepscan, Netherlands

*Correspondence:

Ashraf S. Ibrahim

ibrahim@/undquist.org

Specialty section

This article was submitted to Vaccines and Molecular Therapeutics, a section of the journal

Frontiers in Immunology

Received: 08 October 2019

Accepted: 13 January 2020

Published: 18 February 2020

Citation:

Youssef EG, Zhang L, Alkhazraji S, Gebremariam T, Singh S, Yount NY,

Yeaman MR, Uppuluri $P$ and Ibrahim AS (2020) Monoclonal IgM

Antibodies Targeting Candida albicans

Hyr1 Provide Cross-Kingdom

Protection Against Gram-Negative Bacteria. Front. Immunol. 11:76. doi: 10.3389/fimmu.2020.00076

\section{Monoclonal IgM Antibodies Targeting Candida albicans Hyr1 Provide Cross-Kingdom Protection Against Gram-Negative Bacteria}

\author{
Eman G. Youssef ${ }^{1,2,3}$, Lina Zhang ${ }^{1,4}$, Sondus Alkhazraji ${ }^{1,2}$, Teclegiorgis Gebremariam ${ }^{1,2}$, \\ Shakti Singh ${ }^{1,2}$, Nannette Y. Yount ${ }^{1,5}$, Michael R. Yeaman ${ }^{1,2,5,6}$, Priya Uppuluri ${ }^{1,2,6}$ and \\ Ashraf S. Ibrahim ${ }^{1,2,6 *}$ \\ ${ }^{1}$ Division of Infectious Diseases, Harbor-UCLA Medical Center, Torrance, CA, United States, ${ }^{2}$ The Lundquist Institute for \\ Biomedical Innovation, Harbor-UCLA Medical Center, Torrance, CA, United States, ${ }^{3}$ Department of Biotechnology and Life \\ Sciences, Faculty of Postgraduate Studies for Advanced Sciences, Beni-Suef University, Beni-Suef, Egypt, ${ }^{4}$ College of \\ Wildlife Resources, Northeast Forestry University, Harbin, China, ${ }^{5}$ Division of Molecular Medicine, Harbor-UCLA Medical \\ Center, Torrance, CA, United States, ${ }^{6}$ Department of Medicine, David Geffen School of Medicine at UCLA, Los Angeles, CA, \\ United States
}

Recent years have seen an unprecedented rise in the incidence of multidrug-resistant (MDR) Gram-negative bacteria (GNBs) such as Acinetobacter and Klebsiella species. In view of the shortage of novel drugs in the pipeline, alternative strategies to prevent, and treat infections by GNBs are urgently needed. Previously, we have reported that the Candida albicans hypha-regulated protein Hyr1 shares striking three-dimensional structural homology with cell surface proteins of Acinetobacter baumannii. Moreover, active vaccination with rHyr $1 \mathrm{p}-\mathrm{N}$ or passive immunization with anti-Hyr1p polyclonal antibody protects mice from Acinetobacter infection. In the present study, we use molecular modeling to guide design of monoclonal antibodies (mAbs) generated against Hyr1p and show them to bind to priority surface antigens of Acinetobacter and Klebsiella pneumoniae. The anti-Hyr1 mAbs block damage to primary endothelial cells induced by the bacteria and protect mice from lethal pulmonary infections mediated by $A$. baumannii or K. pneumoniae. Our current studies emphasize the potential of harnessing Hyr1p mAbs as a cross-kingdom immunotherapeutic strategy against MDR GNBs.

Keywords: monoclonal antibodies, Candida Hyr1, Acinetobacter baumannii, Klebsiella pneumoniae, passive vaccine, molecular modeling, cross-kingdom immunotherapy

\section{INTRODUCTION}

Infections caused by multidrug-resistant organisms (MDROs) pose increasing therapeutic challenges. In the past decade, Acinetobacter baumannii has emerged as one of the most common MDROs in hospital-acquired infections, causing a range of diseases from pneumonia to sepsis or wound infections (1-6). Of great concern is that $40-70 \%$ of $A$. baumannii isolates are now extensively drug resistant (XDR; i.e., resistant to all antibiotics except colistin or tigecycline), reflecting a $>15$-fold increase since 2000 (1, 6-8). Likewise, the Enterobacteriaceae organism Klebsiella pneumoniae causes high rates of morbidity and mortality in critically ill, hospitalized patients. In recent years, strains of K. pneumoniae have exhibited resistance to almost all classes 
of antibacterial drugs, including carbapenems (9-11). Together, Acinetobacter and carbapenem-resistant K. pneumoniae (KPC) have been prioritized by the U.S. Centers for Disease Control and Prevention (CDC) as two of the top "serious threat level pathogens" owing to resistance, failure of the current standard of treatment, and high mortality rates. Amplifying these concerns, the existing drug development pipeline against these pathogens is sparse, and it is almost certain that these organisms will develop resistance to any future approved antibiotics. Hence, novel strategies to prevent and treat life-threatening infections caused by these and related MDRO pathogens are urgently needed.

We previously developed innovative computational molecular modeling and bioinformatics strategies to discover novel vaccine and immunotherapy candidates targeting more than one high-priority pathogen. The application of this methodology has been used to successfully discover and develop novel cross-kingdom vaccines (12). Among other advances, this discovery strategy culminated in the identification of Candida albicans Hyrlp, a hypha-regulated cell surface protein. Although Hyrlp is strictly expressed on C. albicans hyphae, it has no effect on the fungus germination and subsequent hyphal formation (13). However, we have shown that Hyrlp contributes to $C$. albicans virulence by resisting phagocyte killing (a major host defense mechanism against candidiasis) through a mechanism that is yet to be identified (14). Indeed, mice vaccinated with Hyrlp are protected from C. albicans infections $(14,15)$. Recently, we found that the Hyrlp shares striking three-dimensional (3-D) structural and epitope homologies with antigens present on the Gram-negative bacterium (GNB) A. baumannii, including with the putative hemagglutinin/hemolysin protein FhaB, outer membrane protein class A (OmpA), and a number of siderophore-binding proteins (16). All these putative cross-reactive antigens are known contributors to bacterial virulence. Specifically, FhaB and OmpA help in bacterial adhesion and biofilm formation (17-19). Also, OmpA plays a role in conferring multidrug resistance of A. baumannii to antibiotics (20). Finally, the A. baumannii siderophore acinetobactin was shown to be required for bacterial infection by acquiring iron from the host (21), therefore implicating siderophore receptors in the virulence of the bacterium. Polyclonal antibodies (pAbs) raised against peptides derived from the Hyrlp N-terminus blocked A. baumannii-mediated lung epithelial cell damage and killed the bacterium in vitro (16). Importantly, anti-Hyrlp pAbs completely protected mice from $A$. baumannii infections. These results provided compelling proof of concept for targeting Hyrlp for developing immunotherapies against GNBs and laid a groundwork for generation and evaluation of the efficacy of anti-Hyrlp monoclonal antibodies (mAbs) targeting MDR GNBs.

In the current study, we generated mAbs against peptide \#5 of Hyr1 and affirm that these mAbs not only recognize different clinical isolates of $A$. baumannii but also bind to drugresistant $K$. pneumoniae. We further demonstrate the efficacy of these targeted mAbs in blocking bacterial-mediated host cell damage and in protecting mice against lethal pulmonary infection by both MDR bacteria. Given that there are currently no immunotherapies against GNBs and the alarming rate at which MDROs are increasing as a global threat to public health, active, or passive vaccination strategies using vaccines or $\mathrm{mAbs}$, respectively, are now highly attractive immunotherapeutic modalities to prevent or treat these refractory infections either as standalone or antibiotic-adjunctive therapies.

\section{RESULTS}

\section{Hyr1p Is Structurally Homologous to Target Surface Antigens of Klebsiella pneumoniae}

Our previous studies involving complimentary homology and energy-based modeling algorithms identified structural domains conserved between Hyrlp and the GNB Acinetobacter baumannii (16). We questioned if other GNBs could similarly share conserved physiochemical structural domains. Of great relevance, we identified strong homology between Hyrlp and filamentous hemagglutinin B (FhaB) of Klebsiella pneumoniae (Figures 1A-D). This highly conserved homology was reflected at the level of amino acid sequences in a shared target motif (Figures 1A-B), relative structural integration of this motif in the larger holoproteins (Figure 1C), and overall 3-D homology of the two proteins (Figure 1D). Furthermore, our modeling studies revealed four other proteins in $K$. pneumoniae that displayed conserved 3-D homology with Hyr1p: OmpA, transporter of nutrients B (TonB), fimbrial protein (Fmp), and the biopolymer export protein $\mathrm{D}(\mathrm{ExbD})$. Following energy minimization and hydrogen-bond optimization to yield 3-D structure models, these proteins were aligned further with a 14-amino-acid peptide of Hyrlp (LKNAVTYDGPVPNN; also called peptide \#5)-a highly antigenic, surface-exposed domain of the protein, for which anti-peptide pAbs were shown to protect against murine A. baumannii infection (16). This was done to localize specific homology sites hypothesized to confer protective efficacy (Figures 1A-D). Modeling data demonstrated that the identified 3-D structures corresponded with a conserved sequence region within each target antigen (Figure 1E). Based on strong efficacy seen in cross-kingdom immunization studies of prior modelingpredicted antigens (e.g., Hyrlp vs. A. baumannii) (16), the current model predictions were interpreted as supporting confidence in cross-protective efficacy against K. pneumoniae.

\section{Anti-Hyr1 Monoclonal Antibodies Bind to Gram-Negative Bacteria}

We previously reported that pAbs raised against Hyr1 peptide \#5 blocked virulence functions of $A$. baumannii in vitro and completely protected diabetic and neutropenic mice from Acinetobacter bacteremia and pulmonary infection, respectively (16). Encouraged by these results, and to enhance the therapeutic potential of such antibodies, we developed mAbs against the same surface-exposed and immunodominant peptide (peptide \#5). These mAbs (all IgM isotypes) were tested for their abilities to bind to Candida albicans as well as the GNB A. baumannii or K. pneumoniae. Four individual fluorescein isothiocyanate (FITC)-labeled mAb clones ( $\mathrm{H} 1, \mathrm{H} 2, \mathrm{H} 3$, and $\mathrm{H} 4 ; 100 \mu \mathrm{g} / \mathrm{ml}$ ) were tested against three prototypic MDR GNB strains, including 


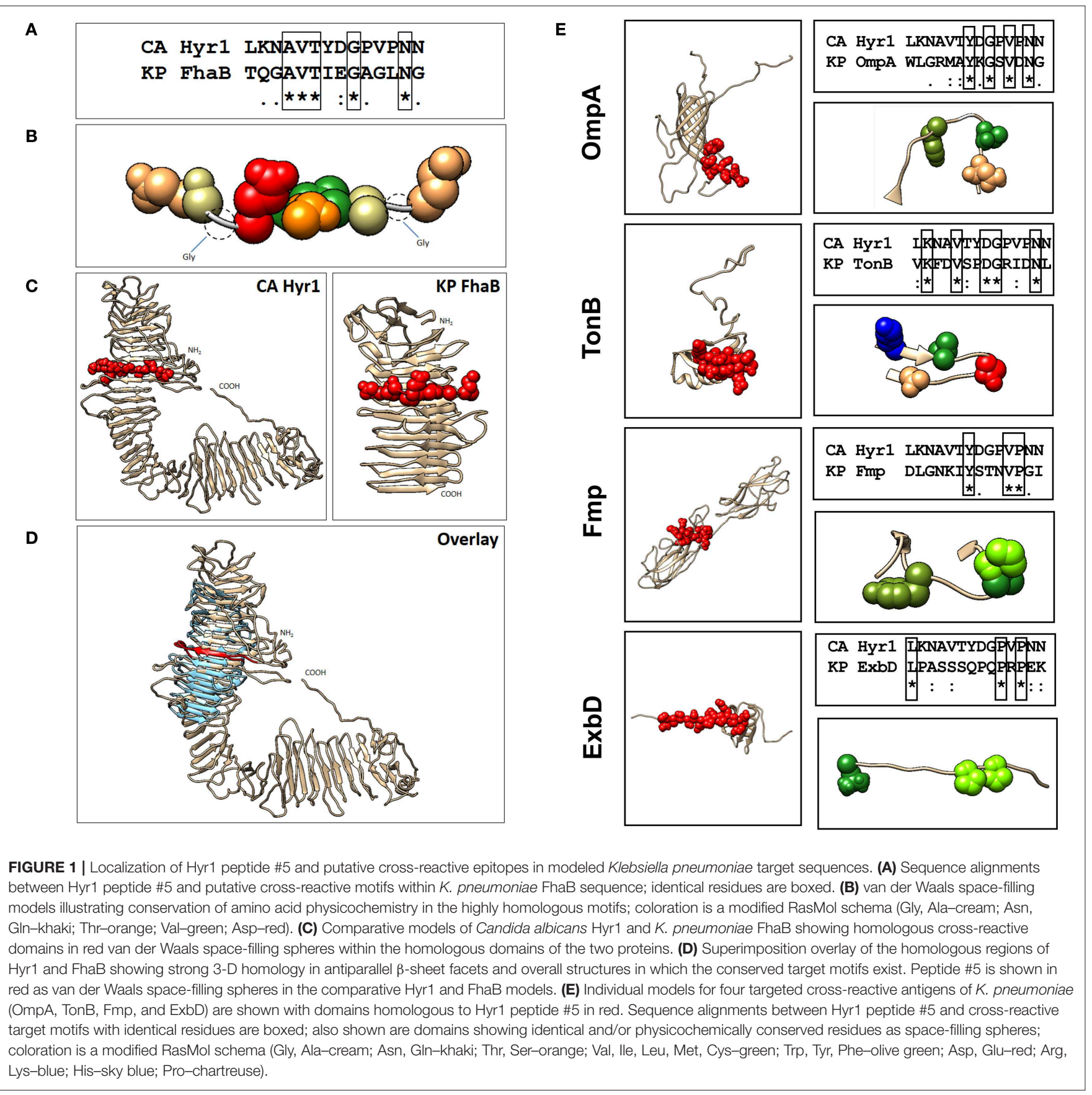

A. baumannii (HUMC-1, XDR clinical isolate); K. pneumoniaeRM (KPC-RM, carbapenem-resistant clinical isolate); and $K$. pneumoniae-QR (KP-QR, MDR strain sensitive to carbapenem). The extent of $\mathrm{mAb}$ binding to each of the pathogen surfaces, as compared with isotype-matched non-specific control antibodies, was then quantified by flow cytometry. Of the four mAb clones tested, compared with the isotype-matched control $\operatorname{IgM}, \mathrm{H} 3$, and $\mathrm{H} 4$ displayed the highest levels of binding to all GNBs, with at least 10-300-fold increases (Figure 2A). Binding potential was also visualized by a shift in the peaks of the anti-Hyrlp IgM binding vs. the isotype-matched control antibodies (Figure 2B).
The right shift in the peaks of individual $\mathrm{mAb}$ also correlated with their respective increase in mean fluorescence of the cells.

Next, we compared the relative binding ability of the $\mathrm{mAb}$ clones to recognize the distinct GNBs. Clone H3 bound to all three organisms even at low mAb concentrations. Specifically, $30 \mu \mathrm{g} / \mathrm{ml}$ of $\mathrm{H} 3 \mathrm{mAb}$ bound 82,75 , and $90 \%$ of A. baumannii HUMC-1, KP-QR, and KPC-RM cells, respectively, whereas the isotype-matched control did not bind to any of the bacterial cells $(<1 \%)$. The binding of the $\mathrm{H} 3 \mathrm{mAbs}$ to either A. baumannii or $K$. pneumoniae was maintained even at a very low concentration of $300 \mathrm{ng} / \mathrm{ml}$, demonstrating 4-10-fold increase over the binding 
A

\begin{tabular}{|l|l|c|c|c|}
\hline \multirow{2}{*}{$\begin{array}{l}\text { Binding } \\
\text { Antibody }\end{array}$} & \multicolumn{3}{|c|}{ Mean fluorescence intensity } \\
\cline { 2 - 5 } & HUMC-1 & KP-QR & KPC-RM \\
\hline $\mathbf{2}$ & IgM Isotype & 287 & 83 & 989 \\
\hline$\square$ & H1 & 15,902 & 5,605 & 6,393 \\
\hline$\square$ & H2 & 12,404 & 3,968 & 6,644 \\
\hline$\square$ & H3 & 54,616 & 10,757 & 8,227 \\
\hline$\square$ & H4 & 31,676 & 14,483 & 9,208 \\
\hline
\end{tabular}

B

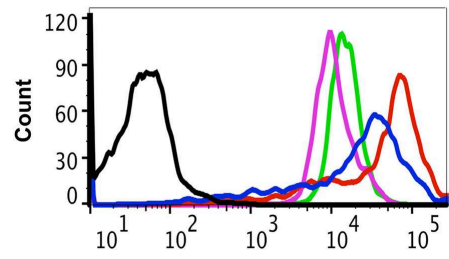

KP-QR

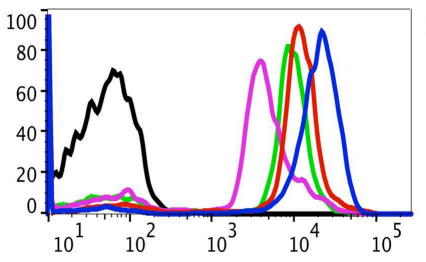

KPC-RM

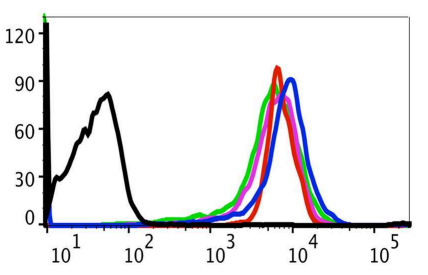

Alexa Fluor 488

FIGURE 2 | Binding of monoclonal antibodies (mAbs) targeting Hyr1 peptide \#5 to Gram-negative bacteria (GNBs). MAb clones (and isotype-matched control) IgM were evaluated for binding to Acinetobacter baumannii (HUMC-1), Klebsiella pneumoniae-QR (KP-QR), and K. pneumoniae-RM (KP-RM) at a concentration of $100 \mu \mathrm{g} / \mathrm{ml}$. The extent of binding was quantified by flow cytometry after staining the bound antibodies with Alexa 488-conjugated secondary antibody. Data were represented as mean fluorescence intensity of the Ab-bound bacteria (A). The degree of binding was also visualized by a shift in the peaks in the anti-Hyr 1 lgM binding conditions vs. the control antibodies (B).

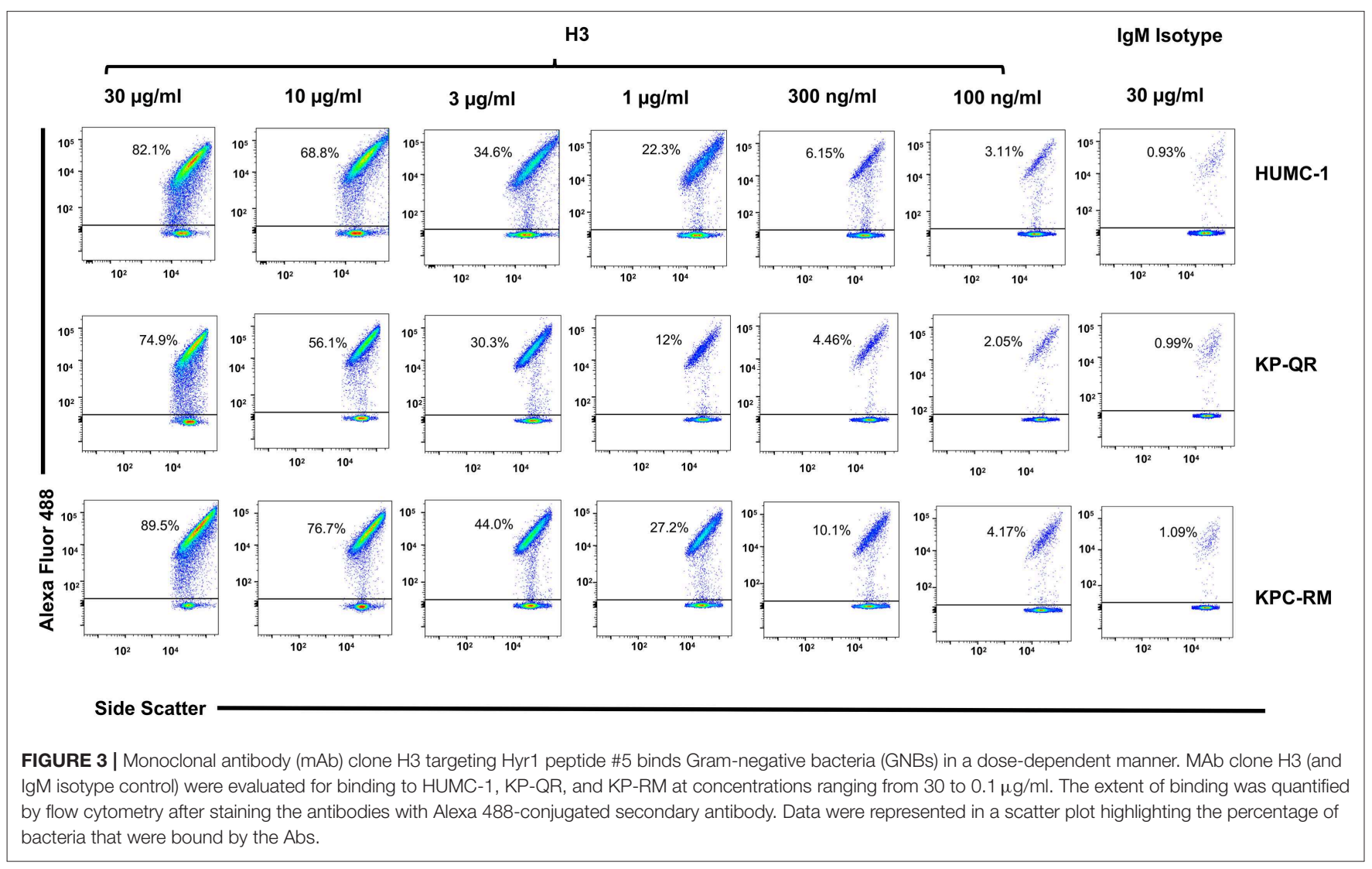

ability of the isotype-matched control IgM (Figure 3). Similarly, the $\mathrm{H} 4$ clone bound to each of the GNBs at $30 \mu \mathrm{g} / \mathrm{ml}$ of concentration (data not shown). We further evaluated the binding of the two clones $\mathrm{H} 3$ and $\mathrm{H} 4$ against other drug-resistant clinical isolates of A. baumannii and K. pneumoniae (KPC). The mAbs bound HUMC-6, HUMC-12, KPC-6, and KPC-8 
at significantly higher capacity as than does the control $\operatorname{IgM}$ (Supplementary Figure 1). These results indicate that binding of mAbs to the surface of the target GNBs is not isolate specific, supporting our consensus epitope hypothesis.

\section{Monoclonal Antibodies Protect Host Cells From Damage by Gram-Negative Bacteria}

Our previous studies demonstrated that anti-Hyrlp pAbs not only bound to A. baumannii but also inhibited the ability of bacterium to interact with and damage mammalian cells (16). Thus, we hypothesized that the mAbs would similarly block damage of host cells caused by these GNBs. Concordant with this hypothesis, $\mathrm{mAb}$ clones $\mathrm{H} 3$ and $\mathrm{H} 4$ at 15 or $30 \mu \mathrm{g} / \mathrm{ml}$ blocked the ability of $A$. baumannii (HUMC-1) and $K$. pneumoniae (KP-QR or KPC-RM) to damage A549 lung alveolar epithelial cells. Specifically, both mAbs showed a dose-response inhibition of GNB-mediated A549 cell damage with the $15 \mu \mathrm{g} / \mathrm{ml}$ of dose resulting in $40-90 \%$ inhibition and the $30 \mu \mathrm{g} / \mathrm{ml}$ dose causing $~ 70-100 \%$ inhibition (Figure 4A). The two mAbs also protected A549 cells from damage by other clinical isolates of GNBs such as HUMC-6 and KPC-8 (Supplementary Figure 2). Consistent with these results, both $\mathrm{mAbs}$ at $15 \mu \mathrm{g} / \mathrm{ml}$ resulted in $\sim 40-70 \%$ inhibition of $A$. baumannii HUMC-1- or KP-QRmediated damage to primary human umbilical vein endothelial cells (HUVECs). However, it took a higher mAb concentration, $30 \mu \mathrm{g} / \mathrm{ml}$, to protect HUVECs from KPC-RM (Figure 4B). Overall, these results show that $\mathrm{mAbs}$ raised against Hyr1 peptide \#5 bind to MDR A. baumannii and K. pneumoniae strains and mitigate the ability of these bacteria to damage host cells in vitro.

\section{Anti-Hyr1 Monoclonal Antibodies Protect Mice From Pulmonary Infection Caused by Acinetobacter baumannii or Klebsiella pneumoniae}

We tested the ability of the mAbs, given their efficacy in reducing GNB-induced host cell damage in vitro, to protect mice from GNB infection. Pneumonia is a life-threatening manifestation of the disease caused by both A. baumannii and K. pneumoniae (22-25). Thus, we evaluated $\mathrm{H} 3$ and $\mathrm{H} 4$ for their ability to protect against such infections caused by $A$. baumannii HUMC-1. Although benign in immunocompetent individuals, A. baumannii can cause life-threatening pneumonia in immunosuppressed hospitalized patients (25). Thus, we additionally evaluated the efficacy of mAb therapy in a neutropenic mouse model infected with HUMC-1 via inhalation. The mAbs were administered intraperitoneally (i.p.) at a dose of $30 \mu \mathrm{g} /$ mouse, in established infection on Days +1 and +4 relative to infection. Placebo mice were treated in an identical regimen with an isotype-matched control IgM. Treatment with mAb $\mathrm{H} 4$ yielded a high (70\%) overall survival, vs. $20 \%$ overall survival for control $\operatorname{IgM}$ treatment $(P<0.06)$. Impressively, complete protection ( $100 \%$ survival) was conferred in mice receiving $\mathrm{H} 3 \mathrm{mAb}$ treatment, $P<0.001$ (Figure 5A). Surviving mice appeared healthy on Day +21 post infection, when the experiment was terminated.
We next evaluated the efficacy of mAbs in a similar mouse model of $K$. pneumoniae pulmonary infection. Our in vivo optimization studies showed that KP-QR exhibits pronounced lethality even in healthy immune competent mice (Supplementary Figure 3A), whereas KPC$\mathrm{RM}$ is avirulent despite high inocula used for infection (Supplementary Figure 3B). Thus, we evaluated the protective effect of mAbs against the KP-QR-mediated pneumonia in mice. Immunocompetent mice were infected intratracheally with KP-QR and treated twice as above with either the $\mathrm{H} 3$ or H4 mAbs, or isotype-matched control IgM. Almost $60 \%$ of mice treated with $\mathrm{H} 4$ survived the otherwise lethal challenge by KP-QR $(P<0.05)$. Consistent with protection against host cell damage, even greater efficacy was observed with $\mathrm{mAb} \mathrm{H3}$, which exhibited protection trending to $80 \%$ survival vs. $20 \%$ survival in mice treated with isotype-matched $\operatorname{IgM}(P<0.05)$ (Figure 5B). Surviving mice appeared healthy at 21 days post infection at the experimental endpoint.

We also performed studies to assess the effect of the mAb treatment on the bacterial burden in lung tissues, along with survival efficacy. Mice were infected as above and treated with $\mathrm{H} 3 \mathrm{mAb}$ once at $6 \mathrm{~h}$ post infection for KP-QR and twice $(6 \mathrm{~h}$ and a repeat dose on Day +3 post infection) for A. baumannii HUMC-1. Mice were sacrificed on Day +2 for KP-QR and Day +4 for HUMC-1, and the lungs were harvested for bacterial burden enumeration by quantitative culture. Corroborating the survival data and in comparison with treatment with isotypematched IgM control, $\mathrm{H} 3 \mathrm{mAb}$ treatment resulted in 1.5- or 3-log reductions in lung bacterial burden of HUMC-1 $(P<0.01)$ or KP-QR $(P<0.001)$, respectively (Figure 5C).

Together, these results demonstrate that therapeutic mAbs derived from innovative methods to exploiting cross-kingdom epitope homology exhibit striking efficacy in life-threating GNB infection. These results emphasize the strong proof-of-concept translational potential to develop such agents as novel therapeutic modalities for prevention or treatment of infections due to MDR GNBs in immunocompetent as well as immunosuppressed or immunodeficient patients.

\section{DISCUSSION}

Phylogenetically diverse pathogens may exploit common host settings and rely on convergent virulence strategies (e.g., cell adhesion, invasion, and injury). Indeed, the fungus Candida albicans and certain GNBs, such as Acinetobacter baumannii and Klebsiella pneumoniae, infect similar immunocompromised, burn, and surgical wound patients in intensive care units (ICUs) or otherwise hospitalized $(7,19,26)$. Interestingly, Candida species colonization among ICU patients have been identified as an independent risk factor for development of $A$. baumannii ventilator-associated pneumonia (27). Similarly, Candida and Klebsiella are the most frequent pathogens of the respiratory tract of patients with chronic obstructive pulmonary disease (COPD) $(28,29)$. Independent of such an association, Candida and GNBs individually cause healthcare-associated infections, often leading to significant morbidity and mortality. As a group, GNBs in 
A

HUMC-1

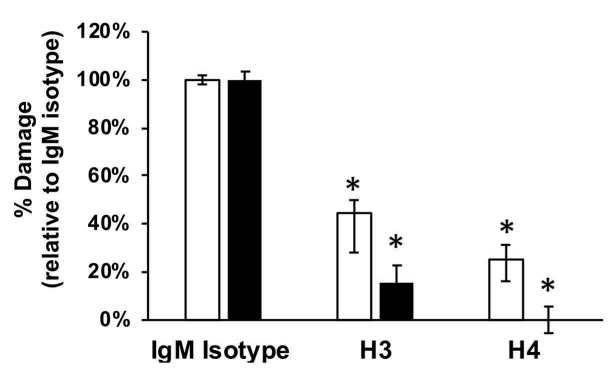

B

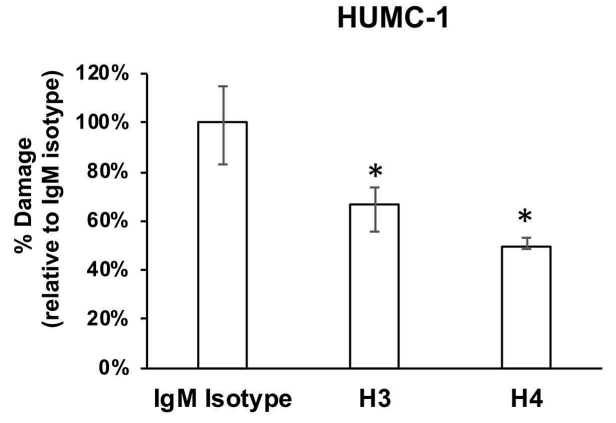

KP-QR

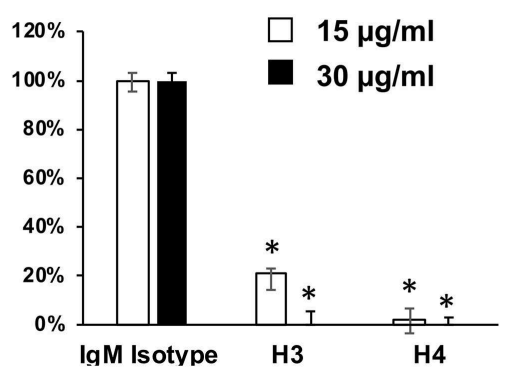

KP-QR

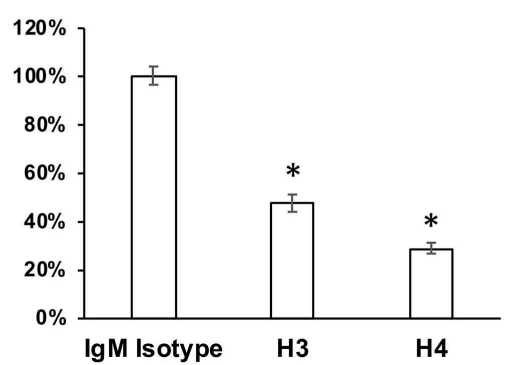

KPC-RM

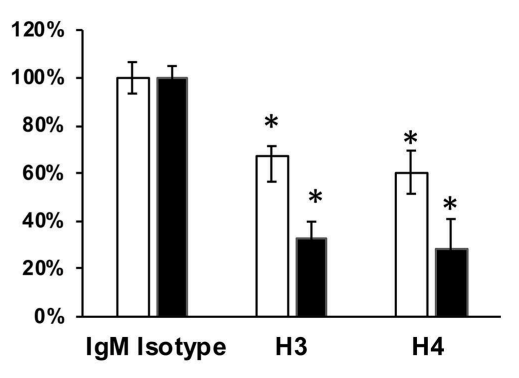

FIGURE 4 | Monoclonal antibodies (mAbs) targeting Hyr1 peptide \#5 prevents HUMC-1-, KP-QR-, and KP-RM-induced A549 lung alveolar epithelial cells and primary human umbilical vein endothelial cells (HUVECs) damage. HUMC-1-, KP-QR-, and KPC-RM-induced A549 cell injury in the presence of 15 or $30 \mu \mathrm{g} / \mathrm{ml}$ of an isotype-matched IgM, mAb H3, or mAb H4 (A). Damage to HUVECs by HUMC-1 and KP-QR (in the presence of $15 \mu \mathrm{g} / \mathrm{ml}$ of $\mathrm{H} 3$ and $\mathrm{H} 4$ ), also $\mathrm{KPC}-\mathrm{RM}$ (30 $\mu \mathrm{g} / \mathrm{ml}$ of the mAbs) (B). Cell damage was determined by ${ }^{51} \mathrm{Cr}$-release assay after 48 and $24 \mathrm{~h}$ for HUMC-1 and KP, respectively. Percentage damage was normalized to lgM isotype-matched control after subtracting spontaneous cell damage. ${ }^{\star} P<0.001 \mathrm{vs}$. control $\operatorname{lgM}$. $N=12$ per group from three independent experiments.

particular, including A. baumannii and K. pneumoniae, have evolved into MDR pathogens that cause infections that are often incurable (30). Hence, novel approaches that address antibiotic resistance and leverage or amplify immune function represent highly logical strategies to combat this resistance crisis.

Our group has developed advanced computational, molecular modeling, and bioinformatics strategies to discover novel vaccine antigen candidates that leverage the concept of convergent immunity to target more than one high-priority human pathogen (16, 31, 32). This strategy, also known as unnatural or heterologous immunity, has been previously applied in the development of viral and bacterial vaccines in which an antigen protects against another pathogen from the same or from a different kingdom $(12,25)$. We have previously validated this approach by demonstrating cross-kingdom immuno-protection against C. albicans and Staphylococcus aureus, in which the C. albicans cell surface adhesin/invasion proteins [agglutinin-like sequence (Als) family of proteins] share epitope and functional homology with MSCRAMMs of S. aureus (e.g., clumping factor A) (33). A recombinant form of $\mathrm{N}$-terminus of the Als3p (rAls $3 \mathrm{p}-\mathrm{N}$ ) elicits robust $\mathrm{T}$ - and $\mathrm{B}$-cell responses and protects mice from both Candida and methicillin-resistant $S$. aureus (MRSA) infections (32, 34-39). Most recently, we reported that a distinct hyphal cell surface protein of C. albicans, Hyrlp, has epitope homologies with candidate antigens of the MDR GNB A. baumannii (16). Indeed, with the use of different mouse models, active or passive immunization (with pAbs) targeting either Als3p or Hyr1p protected mice from S. aureus or A. baumannii infections, respectively $(16,32,34)$. In particular, antibodies against one specific surface-exposed and highly antigenic 15-mer peptide of Hyr1 (peptide \#5) offered the highest protection to host cells from A. baumannii both in vitro and in vivo (16).

Homology and energy-based modeling was conducted to compare the overall and target motif-specific physicochemical features of Hyr1 protein with candidate K. pneumoniae target antigens. These methods predicted Hyrlp to share 3-D and sequence conservation with a number of proteins expressed on the K. pneumoniae surface. In addition to $\mathrm{FhaB}$, significant homologies were observed between the Hyr1 peptide \#5 domain that induced highly protective antisera, and OmpA, TonB, Fmp, and ExbD common to K. pneumoniae and other high-priority GNBs. Encouraged by the potential of the pAbs, and to further the clinical relevance of our studies, we generated mAbs against the highly antigenic peptide \#5 of the Hyr1p. Similar to pAbs, the current results demonstrate that the mAbs blocked the MDR A. baumannii- or K. pneumoniae-mediated host cell damage and protected mice from otherwise lethal pulmonary infections caused by these pathogens. Initial functional assays revealed that four different $\mathrm{mAb}$ clones $(\mathrm{H} 1-\mathrm{H} 4)$ recognized the two genera of bacteria for in vitro binding at low concentrations 


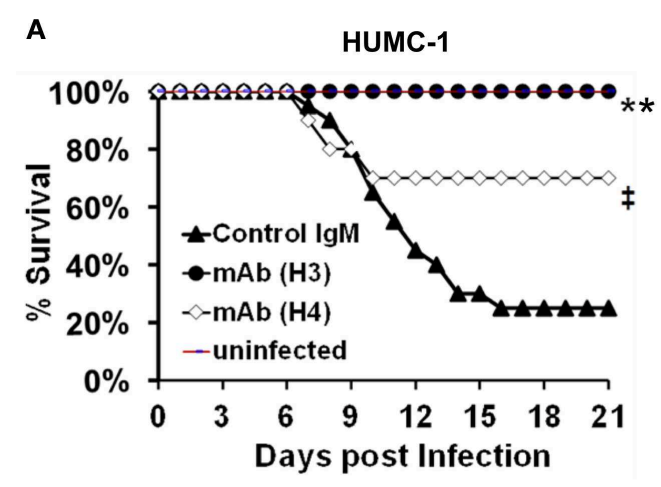

C

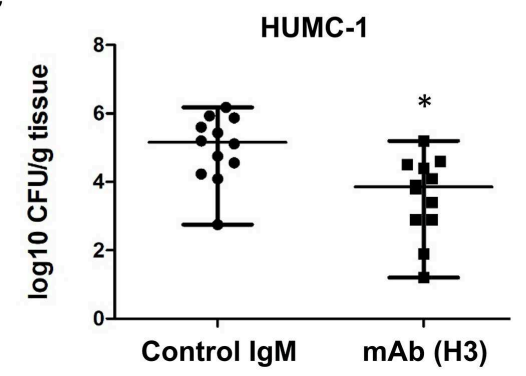

B

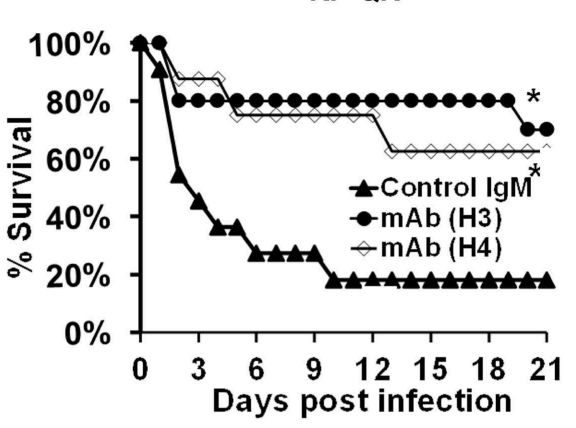

D

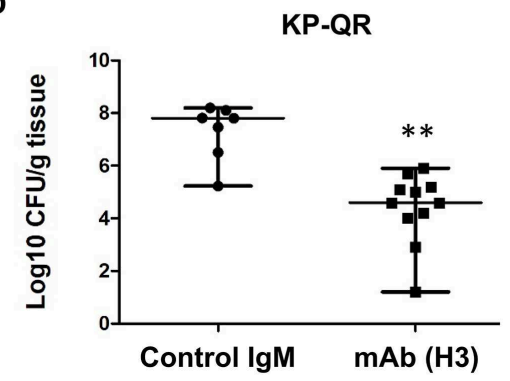

FIGURE 5 | Monoclonal antibody (mAb) clones $\mathrm{H} 3$ and H4, targeting Hyr1 peptide \#5, protect mice from HUMC-1- or KP-QR- induced pneumonia. Immunosuppressed CD-1 male mice ( $n=10$ /group from two experiments) were infected with HUMC-1 via inhalation [average $5 \times 10^{10}$ colony-forming units (CFU)] (A). Immunocompetent mice ( $n=10 /$ group from two experiments) were infected intratracheally with KP-QR (average $\left.3.4 \times 10^{7} \mathrm{CFU}\right)$ (B). Intraperitoneal (i.p.) treatment with $\mathrm{mAb} \mathrm{H} 3, \mathrm{H} 4$, or isotype-matched control lgM started $24 \mathrm{~h}$ and repeated at $96 \mathrm{~h}$ post infection $\left(30 \mu \mathrm{g} / \mathrm{mouse}\right.$ each dose). ${ }^{*} P<0.05, P=0.06$, and ${ }^{* \star} P$ $<0.001$ vs. control lgM by log-rank test. For CFU measurement, $\mathrm{H} 3$ and control lgM were administered $6 \mathrm{~h}$ and 3 days post infection, and lungs harvested from mice at Day +4 (for HUMC-1) (C) and at Day +2 (for KP-QR) post infection (D).

of the antibodies. This targeting propensity was extended also to include several MDR clinical isolates of A. baumannii and K. pneumoniae.

The ability of the mAbs ( $\mathrm{H} 3$ and $\mathrm{H} 4$ ) to block GNB-mediated damage of host cells was more pronounced in A. baumannii HUMC-1, A. baumannii HUMC-6, and K. pneumoniae KPQR than in KPC-RM or KPC-8. Virulence factors, including capsule, lipopolysaccharide, fimbriae, and siderophores, have been identified as important for the virulence and/or resistance of KP strains to antibiotics (40). Thus, the resistance of KPC strains KPC-RM or KPC- 8 to mAbs could conceivably be due to the difference in the exostructure of these organisms [e.g., differences in lipopolysaccharide (LPS) hindrance and reported production of a larger repertoire of siderophores] (40). Consistent with this hypothesis, our recent findings emphasized the importance of anti-Hyr 1 peptide \#5 pAbs in blocking iron uptake, leading to killing of the GNB A. baumannii (16).

Importantly, the mAbs afforded nearly $70 \%$ protection from cellular damage by all GNBs tested, supporting their potential use as preventive or therapeutic modalities with a capacity to block virulence of GNBs. Our recent report using bioinformatics, homology, and energy-based modeling strategies established that $C$. albicans Hyrlp shares striking epitope homology to $A$. baumannii FhaB protein, and anti-peptide $\# 5 \mathrm{pAb}$ bound to FhaB as well as two other proteins on A. baumannii based on two-dimensional Western blotting assays (16). The other two $A$. baumannii proteins with considerable homology to Hyrlp included the OmpA, and a ferric siderophore outer membrane binding protein (TonB) (16). Not surprisingly, these three proteins are well conserved in K. pneumoniae displaying $>60 \%$ sequence homology [protein sequence National Center for Biotechnology Information (NCBI) blast alignment] and even greater 3-D homology to their Acinetobacter counterparts. Whether these proteins have a significant role in virulence or nutrient uptake-and hence blocking their function would contribute to the killing mechanisms afforded by the mAbs-is the subject of ongoing research by our group.

Because the mAbs significantly blocked the capacity of GNBs to damage host cells in vitro, we evaluated their potential to protect against lethal pulmonary infections caused by two prototypic MDR GNBs. In a validated mouse model, mAb H4 afforded $>60 \%$ survival to infection by KP-QR as well as HUMC1 , as compared with control IgM having a $20 \%$ survival rate. Moreover, the mAb $\mathrm{H} 3$ provided $80-100 \%$ survival protection to mice from either of these GNBs, similar to that conferred by pAb (16). The efficacy seen by both $\mathrm{H} 3$ and $\mathrm{H} 4 \mathrm{mAbs}$ is afforded at the low concentration of $30 \mu \mathrm{g} /$ mouse. This low dose of the $\mathrm{mAb}$ is about $1.2 \mathrm{mg} / \mathrm{kg}$, which is within the dosage range of $1-15 \mathrm{mg} / \mathrm{kg}$ of most mAbs approved for human use $(41,42)$. This efficacy suggests that the mAbs likely neutralize functions of specific targets on the bacteria and attenuate their ability to exert virulence mechanisms or to cause disease in the 
host. This hypothesis is further supported by the observation that treatment with $\mathrm{mAbs}$ significantly mitigated dissemination of KP-QR and HUMC-1 to distal target organs within 2-4 days of treatment, vs. mice treated with control antibodies. These results provide compelling evidence of the robustness of the antibodies in abrogating pathogenesis, early in the onset of infection as well as in the setting of established infection.

In addition to specificity and safety, a key advantage of using $\mathrm{mAbs}$ as anti-infective therapy is their well-documented long half-life, which can exceed 21 days $(43,44)$. Because the patient population at risk of developing infections with Acinetobacter, Klebsiella, and Candida are well-defined, this property of mAbs may afford an extended protection during the time span of the greatest vulnerability. In turn, prevention of such infections would translate to reduced use of antibiotics and hence a reduced pressure for the emergence of drug resistance. For example, mAbs can be used to prophylax patients at risk of MDR GNBs. Another envisioned usage of these mAbs is their administration as adjunctive therapy with antibiotics. In this respect, we have demonstrated synergy of anti-peptide \#5 pAb with imipenem or with colistin in killing A. baumannii at reduced minimum inhibitory concentration (MIC) of both antibiotics (16). Similarly, the anti-peptide \#5 pAb synergistically acted with colistin in abrogating biofilm growth of A. baumannii (16).

In theory, one caveat for using novel Abs to treat infections caused by organisms known to develop antimicrobial resistance is the potential development of resistance to these Abs. However, cross-resistance between small molecule antimicrobials and antibacterial mAbs is unlikely because of the distinct therapeutic targets and pharmacological mechanisms that antibodies have as compared with traditional antimicrobials (45). In concept, other potential bacterial defense mechanisms could occur, such as synthesis of antibody-neutralizing proteins (e.g., protein A of $S$. aureus, which binds antibody Fc domain and prevent opsonophagocytosis (46)), or proteases to degrade the administered $\mathrm{mAb}(47,48)$. However, none of the antibodies approved for treating infectious diseases [currently, there are only three Food and Drug Administration (FDA)-approved mAb to treat inhalational anthrax $(49,50)$ or Clostridium difficile $(42)$ have encountered this issue, and development of resistance has not been reported.

In summary, we have demonstrated that mAbs raised against peptide \#5 of Hyr1 target A. baumannii and $K$. pneumoniae and disrupt their ability to damage to host cells in vitro. More importantly, these mAbs protect mice from lethal pulmonary infections mediated by two high-priority GNBs. Thus, such mAbs have credible potential for development as prophylactic or adjunctive therapy to prevent or treat life-threatening infections in patients susceptible to MDR A. baumannii or K. pneumoniae.

\section{MATERIALS AND METHODS}

\section{Bacterial Strains and Growth Conditions}

The bacterial strains used in this study are clinical isolates collected from Harbor-UCLA Medical Center (Torrance, CA). Acinetobacter baumannii strains HUMC-1 and HUMC-6 were separated from patients' sputum, and HUMC-12 was separated from a patient's wound and are XDR to all antibiotics, except colistin and tigecycline. Klebsiella pneumoniae strains were categorized into KPC or non-KPC isolates. The KPC-RM, KPC6 , and KPC-8 isolates resistant to carbapenem antibiotics possess bla KPC plasmid gene and separated from patients' sputum, whereas KP-QR is a non-KPC but multidrug-resistant isolate separated from a patient's sputum and resistant to gentamicin, kanamycin, and ampicillin/sulbactam antibiotics. All bacteria were cultured in tryptic soy broth (TSB) overnight at $37^{\circ} \mathrm{C}$ with shaking at $200 \mathrm{rpm}$. To obtain a log-phase bacterial suspension, overnight cultured bacteria were passaged in a fresh media (1:100) at $37^{\circ} \mathrm{C}$ with shaking for $3 \mathrm{~h}$ or until the cell concentration reached an $\mathrm{OD}_{600}$ of $0.5\left(\sim 2 \times 10^{8}\right.$ cells $\left./ \mathrm{ml}\right)$ for both $A$. baumannii and $K$. pneumoniae isolates. The bacteria were diluted to the desired concentration from this stock.

\section{Computational Modeling of Structural Homology}

Our previously validated Hyrl model (16) was used as a template to seek structural homologs in $K$. pneumoniae having predicted epitopes for cross-kingdom immune protection. The Phyre 2.0 (51) and iTasser (52) platforms were used to generate homology models for Hyr 1 and putative-related proteins. Results were scored based on 3-D threading homology and sequence relatedness and were integrated to identify conserved structural domains. As a confirmatory measure, additional stochastic modeling was carried out using the Quark server (53). Select regions of resulting comparative homologs were then subjected to 3-D alignment to identify areas of greatest homology using the Smith-Waterman (54) algorithm as implemented within Chimera (55). Sequence alignments to identify putative shared epitopes between Hyr 1 and other proteins were carried out using CLUSTALW (56).

\section{Generation of Monoclonal Antibodies}

Thirty micrograms of $\mathrm{rHyr} 1$ peptide \#5 (synthesized by ProMab Biotechnologies, Richmond, CA) in $1 \mathrm{mg} / \mathrm{ml}$ of alum was used to immunize Balb/c mice $(n=10)$. The mice were boosted two times every 2 weeks with the same antigen concentration. Two weeks after the last boost, antibody titer was determined by ELISA plates coated with rHyr1 peptide \#5. The spleens were collected, and the splenocytes were fused with hypoxanthineguanine phosphoribosyltransferase (HGPRT)-negative murine myeloma cells at ratio 5:1 by slowly adding polyethylene glycol (PEG) to the cells pellet followed by adding Protein Free Hybridoma Media (PFHM) (Gibco, 12040077) supplemented with $20 \%$ heat-inactivated fetal bovine serum (FBS) (Corning, $35-016-\mathrm{CV})$. The cells were spun and re-suspended in $20 \%$ FBS $\mathrm{PFHM}$ and then incubated in 24 -well plate at $37^{\circ} \mathrm{C}$ with $5 \%$ $\mathrm{CO}_{2}$ for $48 \mathrm{~h}$. The media were replaced with hypoxanthineaminopterin-thymidine (HAT) selection media for 8 days and then 20\% FBS PFHM hypoxanthine-thymidine (HT) media for 2 weeks. The grown hybridoma clones were diluted by microdilution in microtiter plates to achieve one cell per well and propagated in 10\% FBS PFHM. The supernatant from the grown clones was tested for anti-Hyr1 antibodies using ELISA. The selected positive and stable clones were cultured in PFHM 
without FBS, and the cell numbers were adjusted to be $2 \times 10^{5} / \mathrm{ml}$ for optimum production of mAbs.

\section{Detoxification and Purification of the Supernatant Containing the Monoclonal Antibodies}

The supernatant containing the antibodies was concentrated using $100-\mathrm{kDa}$ cutoff centrifugal concentrating tube (Amicon, UFC910024). HiTrap HP column (GE Healthcare, 17511001) was used to purify the concentrated mAbs and then was buffer exchanged with endotoxin-free Dulbecco phosphatebuffered saline (PBS) without calcium or magnesium (Gibco, 14190250). Endotoxin was tested using a chromogenic limulus amebocyte lysate assay (BioWhittaker Inc.), and all mAbs had low-range endotoxin level of $<0.015 \mathrm{EU} / \mathrm{ml}$. The isotype of the mAbs was identified using ELISA and confirmed by molecular weight using sodium dodecyl sulfate-polyacrylamide gel electrophoresis (SDS-PAGE).

\section{Surface Staining and Binding Assay}

Bacterial cells $\left(5 \times 10^{6}\right.$ cells $)$ re-suspended in $2 \%$ FBS-PBS were incubated with anti-Hyr $1 \mathrm{mAbs}$ or isotype-matched IgM control (BD Biosciences) for $2 \mathrm{~h}$ at a range of concentrations $(100-0.1 \mu \mathrm{g} / \mathrm{ml})$. The bacterial cells were washed three times with cold $2 \%$ FBS-PBS. The bound anti-Hyr1 mAbs to the bacterial cells were detected with anti-mouse FITC-labeled secondary antibodies (Thermo Fisher Scientific). The unbound antibodies were washed three times with cold 2\% FBS-PBS before the measurement of the fluorescent-stained bacterial cells using flow cytometry (Becton Dickinson FACSCalibur), where it is adjusted to detect up to 20,000 events per sample.

\section{Cell Damage Assay}

The in vitro ability of mAbs to protect either A549 cells or HUVECs from damage caused by direct contact with bacteria was measured using ${ }^{51} \mathrm{Cr}$ release assay, modified from previous method (57). Isolation of HUVECs was performed in the laboratory under a protocol approved by institutional review board (IRB). Because umbilical cords are collected without donor identifiers, our IRB considers them medical waste and not subject to informed consent.

Alveolar epithelial A549 cells and HUVECs were incubated overnight in 24-well plates with F-12K or Roswell Park Memorial Institute (RPMI1640) medium supplemented with 10\% FBS, containing $1 \mu \mathrm{Ci}$ /well of $\mathrm{Na}_{2}{ }^{51} \mathrm{CrO}_{4}$ (ICN Biomedicals, Irvine, CA). The next day, unincorporated tracer was aspirated, and the wells were rinsed three times with warm Hanks' Balanced Salt Solution (HBSS). One milliliter of media containing HUMC1 or KP-QR (pre-incubated with mAbs or IgM isotype control for $1 \mathrm{~h}$ on ice) was then added to host cells in each well at a multiplicity of infection (MOI) of 1:100 (host cells to bacteria), and the plate was incubated for 48 or $24 \mathrm{~h}$, respectively, at $37^{\circ} \mathrm{C}$ in $5 \% \mathrm{CO}_{2}$. At the end of the incubation, all the media were gently aspirated from each well, after which the mammalian cells were lysed by the addition of $0.5 \mathrm{ml}$ of $6 \mathrm{~N} \mathrm{NaOH}$. The lysed cells were aspirated, and the wells were rinsed twice with RadioWash (Atomic Products, Inc., Shirley, NY). These rinses were added to the lysed cells, and the ${ }^{51} \mathrm{Cr}$ radioactivity of the medium and the cell lysates was determined. Control wells containing media but no organisms were processed in parallel to measure the spontaneous release of ${ }^{51} \mathrm{Cr}$. After corrections were made for the differences in the incorporation of ${ }^{51} \mathrm{Cr}$ in each well, the specific release of ${ }^{51} \mathrm{Cr}$ was calculated by the following formula: (experimental release-spontaneous release)/(total incorporation-spontaneous release).

\section{Animal Models}

Male CD-1 immunocompetent mice (4-6 weeks old) were used for Klebsiella (KP-QR) intratracheal infection or immunosuppressed mice infected with A. baumannii (HUMC1) by an aerosolization chamber to induce pneumonia by inhalation. Mice were immunosuppressed by administrating cyclophosphamide $(200 \mathrm{mg} / \mathrm{kg})$ (i.p.) and cortisone acetate $(250$ $\mathrm{mg} / \mathrm{kg}$ ) (subcutaneous) on Days $-2,+3$, and +8 relative to infection as previously described Gebremariam et al. (57). A total of $30 \mu \mathrm{g} / \mathrm{mouse}$ of mAbs or isotype-matched control were administrated (i.p.) on Day +1 and on Day +4 post infection. Survival of mice served as an endpoint. For quantitative measurement of bacterial burden, mAbs were administered $6 \mathrm{~h}$ after infection, and a repeat dose was given on Day +3 . Mice were euthanized on Day +4 for A. baumannii and on Day +2 for $K$. pneumoniae. Lungs were harvested aseptically and homogenized, and the bacterial burden was determined by quantitative culturing on tryptic soy agar plates.

\section{Statistical Analysis}

The percentage of cell damage and tissue bacterial burden was compared using non-parametric Mann-Whitney test. The logrank test was used to determine the difference in survival studies. $P<0.05$ was considered significant.

\section{DATA AVAILABILITY STATEMENT}

All datasets generated for this study are included in the article/Supplementary Material.

\section{ETHICS STATEMENT}

All procedures involving mice were approved by the Institutional Animal Care and Use Committee (IACUC) of the Lundquist Institute for Biomedical Innovation at Harbor-UCLA Medical Center (protocol number 20295), according to the National Institutes of Health (NIH) guidelines for animal housing and care. Moribund mice according to detailed and wellcharacterized criteria were euthanized by pentobarbital overdose, followed by cervical dislocation.

\section{AUTHOR CONTRIBUTIONS}

EY performed conceptualization, data curation, formal analysis, investigation, methodology, and writing-original draft. SA, TG, and LZ performed investigation and methodology. SS performed data analysis and manuscript revision. NY performed methodological and data interpretation. MY performed conceptualization, methodology, data interpretation, and manuscript revision. PU performed formal analysis, 
investigation, methodology, and writing-original draft. AI performed conceptualization, data curation, formal analysis, funding acquisition, investigation, methodology, project administration, supervision, and manuscript revision.

\section{FUNDING}

This work was supported by NIH grants R33 AI119339 and 1R01AI141202-01 to AI. The funders had no role in study design, data collection and analysis, decision to publish, or preparation of the manuscript.

\section{REFERENCES}

1. Perez F, Hujer AM, Hujer KM, Decker BK, Rather PN, Bonomo RA. Global challenge of multidrug-resistant Acinetobacter baumannii. Antimicrob Agents Chemother. (2007) 51:3471-84. doi: 10.1128/AAC.01464-06

2. Falagas ME, Karveli EA, Siempos II, Vardakas KZ. Acinetobacter infections, a growing threat for critically ill patients. Epidemiol Infect. (2008) 136:100919. doi: $10.1017 /$ S0950268807009478

3. Karageorgopoulos DE, Falagas ME. Current control and treatment of multidrug-resistant Acinetobacter baumannii infections. Lancet Infect Dis. (2008) 8:751-62. doi: 10.1016/S1473-3099(08)70279-2

4. Higgins PG, Dammhayn C, Hackel M, Seifert H. Global spread of carbapenem-resistant Acinetobacter baumannii. J Antimicrob Chemother. (2009) 65:233-8. doi: 10.1093/jac/dkp428

5. Doi Y, Husain S, Potoski BA, McCurry KR, Paterson DL. Extensively drug-resistant Acinetobacter baumannii. Emerg Infect Dis. (2009) 15:9802. doi: $10.3201 /$ eid1506.081006

6. Hoffmann MS, Eber MR, Laxminarayan R. Increasing resistance of acinetobacter species to imipenem in United States hospitals, 19992006. Infect Control Hosp Epidemiol. (2009) 31:196-7. doi: 10.1086/6 50379

7. Rosenthal VD, Maki DG, Jamulitrat S, Medeiros EA, Todi SK, Gomez DY, et al. International Nosocomial Infection Control Consortium (INICC) report, data summary for 2003-2008, issued June 2009. Am J Infect Control. (2010) 38:95-104.e2. doi: 10.1016/j.ajic.2009.12.004

8. Lautenbach E, Synnestvedt M, Weiner MG, Bilker WB, Vo L, Schein J, et al. Epidemiology and impact of imipenem resistance in Acinetobacter baumannii. Infect Control Hosp Epidemiol. (2009) 30:1186-92. doi: 10.1086/648450

9. Mammina C, Bonura C, Di Bernardo F, Aleo A, Fasciana T, Sodano C, et al. Ongoing spread of colistin-resistant Klebsiella pneumoniae in different wards of an acute general hospital, Italy, June to December 2011. Euro Surveill. (2012) 17:20248.

10. Brink AJ, Coetzee J, Corcoran C, Clay CG, Hari-Makkan D, Jacobson RK, et al. Emergence of OXA-48 and OXA-181 carbapenemases among Enterobacteriaceae in South Africa and evidence of in vivo selection of colistin resistance as a consequence of selective decontamination of the gastrointestinal tract. J Clin Microbiol. (2013) 51:369-72. doi: 10.1128/JCM.02234-12

11. Giordano C, Barnini S, Tsioutis C, Chlebowicz MA, Scoulica EV, Gikas A, et al. Expansion of KPC-producing Klebsiella pneumoniae with various mgrBmutations giving rise to colistin-resistance, the role of ISL3 on plasmids. Int J Antimicrob Agents. (2017) 51:260-5. doi: 10.1016/j.ijantimicag.2017. 10.011

12. Yeaman MR, Hennessey JP. Innovative approaches to improve antiinfective vaccine efficacy. Annu Rev Pharmacol Toxicol. (2017) 57:189222. doi: 10.1146/annurev-pharmtox-010716-104718

13. Bailey DA, Feldmann PJ, Bovey M, Gow NA, Brown AJ. The Candida albicans HYR1 gene, which is activated in response to hyphal development, belongs to a gene family encoding yeast cell wall proteins. J Bacteriol. (1996) 178:535360. doi: 10.1128/JB.178.18.5353-5360.1996

\section{ACKNOWLEDGMENTS}

We thank Sameh Soliman and Wessam Abdelhady for the valuable discussions. This manuscript has been released as a pre-print at bioRxiv (58).

\section{SUPPLEMENTARY MATERIAL}

The Supplementary Material for this article can be found online at: https://www.frontiersin.org/articles/10.3389/fimmu. 2020.00076/full\#supplementary-material

14. Luo G, Ibrahim AS, Spellberg B, Nobile CJ, Mitchell AP, Fu Y. Candida albicans Hyrlp confers resistance to neutrophil killing and is a potential vaccine target. J Infect Dis. (2010) 201:1718-28. doi: 10.1086/652407

15. Luo G, Ibrahim AS, French SW, Edwards JE, Fu Y. Active and passive immunization with rHyrlp-N protects mice against hematogenously disseminated candidiasis. PLoS ONE. (2011) 6:e25909. doi: 10.1371/journal.pone.0025909

16. Uppuluri P, Lin L, Alqarihi A, Luo G, Youssef EG, Alkhazraji S, et al. The Hyrl protein from the fungus Candida albicans is a cross kingdom immunotherapeutic target for Acinetobacter bacterial infection. PLoS Pathog, (2018) 14:e1007056. doi: 10.1371/journal.ppat.1007056

17. Darvish Alipour Astaneh S, Rasooli I, Mousavi Gargari SL. The role of filamentous hemagglutinin adhesin in adherence and biofilm formation in Acinetobacter baumannii ATCC19606. Microb Pathog. (2014) 74(Supplement C):42-9. doi: 10.1016/j.micpath.2014.07.007

18. Darwish Alipour Astaneh S, Rasooli I, Mousavi Gargari SL. Filamentous hemagglutinin adhesin FhaB limits A. baumannii biofilm formation. Front Biosci. (2017) 9:266-75. doi: 10.2741/e801

19. Gaddy JA, Tomaras AP, Actis LA. The Acinetobacter baumannii 19606 OmpA protein plays a role in biofilm formation on abiotic surfaces and in the interaction of this pathogen with eukaryotic cells. Infect Immun. (2009) 77:3150-60. doi: 10.1128/IAI.00096-09

20. Smani Y, Fàbrega A, Roca I, Sánchez-Encinales V, Vila J, Pachón J. Role of OmpA in the multidrug resistance phenotype of Acinetobacter baumannii. Antimicrob Agents Chemother. (2014) 58:1806-8. doi: 10.1128/AAC.02101-13

21. Gaddy JA, Arivett BA, McConnell MJ, López-Rojas R, Pachón J, Actis LA. Role of acinetobactin-mediated iron acquisition functions in the interaction of Acinetobacter baumannii strain ATCC 19606T with human lung epithelial cells, Galleria mellonella caterpillars, and mice. Infect Immun. (2012) 80:101524. doi: 10.1128/IAI.06279-11

22. Trouillet JL, Chastre J, Vuagnat A, Joly-Guillou ML, Combaux D, Dombret MC, et al. Ventilator-associated pneumonia caused by potentially drug-resistant bacteria. Am J Respir Crit Care Med. (1998) 157:5319. doi: 10.1164/ajrccm.157.2.9705064

23. Park DR. The microbiology of ventilator-associated pneumonia. Respir Care. (2005) 50:742-63; discussion 763-5.

24. American Thoracic Society; Infectious Diseases Society of America Guidelines for the management of adults with hospital-acquired, ventilatorassociated, and healthcare-associated pneumonia. Am J Respir Crit Care Med. (2005) 171:388-416. doi: 10.1164/rccm.200405-644ST

25. Caricato A, Montini L, Bello G, Michetti V, Maviglia R, Bocci $\mathrm{MG}$, et al. Risk factors and outcome of Acinetobacter baumanii infection in severe trauma patients. Intensive Care Med. (2009) 35:1964-9. doi: 10.1007/s00134-009-1582-5

26. Peleg AY, Seifert H, Paterson DL. Acinetobacter baumannii, emergence of a successful pathogen. Clin Microbiol Rev. (2008) 21:538-82. doi: 10.1128/CMR.00058-07

27. Tan X, Zhu S, Yan D, Chen W, Chen R, Zou J, et al. Candida spp. airway colonization, a potential risk factor for Acinetobacter baumannii ventilator-associated pneumonia. Med Mycol. (2016) 54:557-66. doi: 10.1093/mmy/myw009 
28. Przybylowska D, Piskorska K, Golas M, Sikora M, Swoboda-Kopec E, Kostrzewa-Janicka J, et al. Evaluation of genetic diversity of Candida spp. and Klebsiella spp. isolated from the denture plaque of COPD patients. Adv Exp Med Biol. (2017) 955:1-8. doi: 10.1007/5584_2016_68

29. Su J, Liu HY, Tan XL, Ji Y, Jiang YX, Prabhakar M, et al. Sputum bacterial and fungal dynamics during exacerbations of severe COPD. PLoS ONE. (2015) 10:e0130736. doi: 10.1371/journal.pone.0130736

30. Ventola CL. The antibiotic resistance crisis, part 1 , causes and threats. $P T$, (2015) 40:277-83. doi: 10.1055/s-0035-1552326

31. Yeaman MR, Filler SG, Schmidt CS, Ibrahim AS, Edwards JE, Hennessey JP. Applying convergent immunity to innovative vaccines targeting Staphylococcus aureus. Front Immunol. (2014) 5:463. doi: 10.3389/fimmu.2014.00463

32. Yeaman MR, Filler SG, Chaili S, Barr K, Wang H, Kupferwasser $\mathrm{D}$, et al. Mechanisms of NDV-3 vaccine efficacy in MRSA skin versus invasive infection. Proc Natl Acad Sci USA. (2014) 111:E5555-63. doi: 10.1073/pnas.1415610111

33. Sheppard DC, Yeaman MR, Welch WH, Phan QT, Fu Y, Ibrahim AS, et al. Functional and structural diversity in the Als protein family of Candida albicans. J Biol Chem. (2004) 279:30480-9. doi: 10.1074/jbc.M401929200

34. Lin L, Ibrahim AS, Xu X, Farber JM, Avanesian V, Baquir B, et al. Th1Th17 cells mediate protective adaptive immunity against Staphylococcus aureus and Candida albicans infection in mice. PLoS Pathog. (2009) 5:e1000703. doi: 10.1371/journal.ppat.1000703

35. Spellberg B, Ibrahim AS, Yeaman MR, Lin L, Fu Y, Avanesian V, et al. The antifungal vaccine derived from the recombinant $\mathrm{N}$ terminus of Als3p protects mice against the bacterium Staphylococcus aureus. Infect Immun. (2008) 76:4574-80. doi: 10.1128/IAI.00700-08

36. Lin L, Ibrahim AS, Baquir B, Avanesian V, Fu Y, Spellberg B. Immunological surrogate marker of $\mathrm{rAls} 3 \mathrm{p}-\mathrm{N}$ vaccine-induced protection against Staphylococcus aureus. FEMS Immunol Med Microbiol. (2009) 55:293-5. doi: 10.1111/j.1574-695X.2008.00531.x

37. Spellberg B, Ibrahim AS, Lin L, Avanesian V, Fu Y, Lipke P, et al. Antibody titer threshold predicts anti-candidal vaccine efficacy even though the mechanism of protection is induction of cell-mediated immunity. J Infect Dis. (2008) 197:967-71. doi: 10.1086/529204

38. Yeaman M, Filler S, Chaili S, Barr K, Wang H, Kupferwasser D, et al. Efficacy and immunologic mechanisms of NDV-3 vaccine in a murine model of methicillin-resistant Staphylococcus aureus (MRSA) skin/skin structure infection (SSSI). In: 52nd Interscience Conference on Antimicrobial Agents and Chemotherapy. San Francisco, CA (2012)

39. Yeaman MR, Ibrahim AS, Filler SG, Chaili S, Barr K, Wang H, et al. Efficacy of NDV3 vaccine in a murine model of Staphylococcus aureus skin/soft tissue infection (SSTI). In: Gordon Research Conference on Staphylococcal Diseases. Barga, Italy (2011).

40. Paczosa MK, Mecsas J. Klebsiella pneumoniae, going on the offense with a strong defense. Microbiol Mol Biol Rev. (2016) 80:629-61. doi: 10.1128/MMBR.00078-15

41. Hendrikx JJMA, Haanen JBAG, Voest EE, Schellens JHM, Huitema ADR, Beijnen JH. Fixed dosing of monoclonal antibodies in oncology. Oncologist. (2017) 22:1212-21. doi: 10.1634/theoncologist.2017-0167

42. Wilcox MH, Gerding DN, Poxton IR, Kelly C, Nathan R, Birch T, et al. Bezlotoxumab for prevention of recurrent clostridium difficile infection. $N$ England J Med. (2017) 376:305-17. doi: 10.1056/NEJMoa1602615

43. Curtin F, Lang AB, Perron H, Laumonier M, Vidal V, Porchet $\mathrm{HC}$, et al. GNbAC1, a humanized monoclonal antibody against the envelope protein of multiple sclerosis-associated endogenous retrovirus, a first-in-humans randomized clinical study. Clin Ther. (2012) 34:2268-78. doi: 10.1016/j.clinthera.2012.11.006

44. Xu L, Lu T, Tuomi L, Jumbe N, Lu J, Eppler S, et al. Pharmacokinetics of ranibizumab in patients with neovascular age-related macular degeneration, a population approach. Invest Ophthalmol Vis Sci. (2013) 54:161624. doi: 10.1167/iovs.12-10260

45. Wang-Lin SX, Balthasar JP. Pharmacokinetic and pharmacodynamic considerations for the use of monoclonal antibodies in the treatment of bacterial infections. Antibodies. (2018) 7:5. doi: 10.3390/antib70 10005

46. Falugi F, Kim HK, Missiakas DM, Schneewind O. Role of protein A in the evasion of host adaptive immune responses by Staphylococcus aureus. mBio. (2013) 4:e00575. doi: 10.1128/mBio.00575-13

47. von Pawel-Rammingen U, Johansson BP, Björck L. IdeS, a novel streptococcal cysteine proteinase with unique specificity for immunoglobulin G. EMBO J. (2002) 21:1607-15. doi: 10.1093/emboj/21.7.1607

48. Fick RB, Baltimore RS, Squier SU, Reynolds HY. IgG proteolytic activity of Pseudomonas aeruginosa in cystic fibrosis. J Infect Dis. (1985) 151:58998. doi: 10.1093/infdis/151.4.589

49. Chen Z, Moayeri M, Purcell R. Monoclonal antibody therapies against anthrax. Toxins. (2011) 3:1004-19. doi: 10.3390/toxins3081004

50. Nagy CF, Mondick J, Serbina N, Casey LS, Carpenter SE, French J, et al. Animal-to-human dose translation of obiltoxaximab for treatment of inhalational anthrax under the US FDA animal rule. Clin Transl Sci. (2017) 10:12-9. doi: 10.1111/cts.12433

51. Kelley LA, Mezulis S, Yates CM, Wass MN, Sternberg MJ. The Phyre2 web portal for protein modeling, prediction and analysis. Nat Protoc. (2015) 10:845-58. doi: 10.1038/nprot.2015.053

52. Yang J, Yan R, Roy A, Xu D, Poisson J, Zhang Y. The I-TASSER suite, protein structure and function prediction. Nat Methods. (2015) 12:78. doi: $10.1038 /$ nmeth.3213

53. $\mathrm{Xu} \mathrm{D}$, Zhang $\mathrm{Y}$. Ab initio protein structure assembly using continuous structure fragments and optimized knowledge-based force field. Proteins. (2012) 80:1715-35. doi: 10.1002/prot.24065

54. Smith TF, Waterman MS. Identification of common molecular subsequences. J Mol Biol. (1981) 147:195-7. doi: 10.1016/0022-2836(81)90087-5

55. Pettersen EF, Goddard TD, Huang CC, Couch GS, Greenblatt DM, Meng EC, et al. UCSF Chimera-a visualization system for exploratory research and analysis. J Comput Chem. (2004) 25:1605-12. doi: 10.1002/jcc.20084

56. Higgins DG, Sharp PM. CLUSTAL, a package for performing multiple sequence alignment on a microcomputer. Gene. (1988) 73:237-44. doi: 10.1016/0378-1119(88)90330-7

57. Gebremariam T, Liu M, Luo G, Bruno V, Phan QT, Waring AJ, et al. CotH3 mediates fungal invasion of host cells during mucormycosis. J Clin Invest. (2014) 124:237-50. doi: 10.1172/JCI71349

58. Youssef E, Alkhazraji S, Gebremariam T, Zhang L, Singh S, Yount N, et al. Monoclonal IgM antibodies raised against Candida albicans. Hyr1 provide cross-kingdom protection against Gram negative bacteria. bioRxiv. (2019). doi: 10.1101/687442

Conflict of Interest: MY and AI are founders and shareholders of NovaDigm Therapeutics, Inc., which is developing novel immunotherapies targeting priority pathogens.

The remaining authors declare that the research was conducted in the absence of any commercial or financial relationships that could be construed as a potential conflict of interest.

Copyright (c) 2020 Youssef, Zhang, Alkhazraji, Gebremariam, Singh, Yount, Yeaman, Uppuluri and Ibrahim. This is an open-access article distributed under the terms of the Creative Commons Attribution License (CC BY). The use, distribution or reproduction in other forums is permitted, provided the original author(s) and the copyright owner(s) are credited and that the original publication in this journal is cited, in accordance with accepted academic practice. No use, distribution or reproduction is permitted which does not comply with these terms. 